\title{
Motorcycle Movement Model Based on Markov Chain Process in Mixed Traffic
}

\author{
Rina Mardiati $^{1}$, Bambang R. Trilaksono ${ }^{2}$, Yudi S. Gondokaryono ${ }^{3}$, and Sony S. Wibowo ${ }^{4}$ \\ ${ }^{1,2,3}$ School of Electrical Engineering and Informatics, Bandung Institute of Technology, Indonesia \\ ${ }^{4}$ Department of Civil Engineering, Bandung Institute of Technology, Indonesia
}

\section{Article Info}

Article history:

Received August 9, 2017

Revised June 25, 2018

Accepted July 7, 2018

\section{Keyword:}

Markov Chain

Maneuver Intention

Vehicle Movement

Mixed Traffic

\begin{abstract}
Mixed traffic systems are dynamically complex since there are many parameters and variables that influence the interactions between the different kinds of vehicles. Modeling the behavior of vehicles, especially motorcycle which has erratic behavior is still being developed continuously, especially in developing countries which have heterogeneous traffic. To get a better understanding of motorcycle behavior, one can look at maneuvers performed by drivers. In this research, we tried to build a model of motorcycle movement which only focused on maneuver action to avoid the obstacle along with the trajectories using a Markov Chain approach. In Markov Chain, the maneuver of motorcycle will described by state transition. The state transition model is depend on probability function which will use for determine what action will be executed next. The maneuver of motorcycle using Markov Chain model was validated by comparing the analytical result with the naturalistic data, with similarity is calculated using MSE. In order to know how good our proposed method can describe the maneuver of motorcycle, we try to compare the MSE of the trajectory based on Markov Chain model with those using polynomial approach. The MSE results showed the performance of Markov Chain Model give the smallest MSE which 0.7666 about 0.24 better than $4^{\text {th }}$ order polynomial.
\end{abstract}

Copyright (c) 2018 Institute of Advanced Engineering and Science. All rights reserved.

Corresponding Author:

Rina Mardiati

Department of Electrical Engineering, UIN Sunan Gunung Djati Bandung

Jl. A.H. Nasution No. 105 Bandung 40614, Indonesia

r_mardiati@uinsgd.ac.id

\section{INTRODUCTION}

Today, Indonesian transportation problems are increasingly being encountered in our daily life. Several economic and social motivations can be related to the need to minimize the time spent in motorcycle for transportation and consequently their related pollution problems. An additional problem worth mentioning is the need to reduce traffic accidents, a human and social cost that is related not only inadequate driving, but also to the planning of the flow conditions [1]. Due this motivations, the literature on traffic phenomena is already vast and characterized by contributions covering modeling aspects, statement of problems, qualitative analysis, and particularly developed simulation generated by applications. Continuing this efforts, there are many literature of traffic flow theories and models have been developed, but researcher generally agree that modeling has not yet reached a satisfying level. Study of traffic flow are become important since many reasons behind that, such as: 1) it is necessary to develop traffic model which can describes the real phenomena, 2) traffic model can support for developing intelligent transportation system (ITS) whose related with safety driving system, 3) traffic model support for future issue about intelligent car [2]. So, there are many researchers who developed intelligent transportation system in order to minimize the number of traffic accident [3] [4] [5].

Mixed traffic systems are dynamically complex since there are many parameters and variables that influence the interactions between the different kinds of motorcycles. These interactions can be described as the behavior occurring in traffic. Vehicle behavior is influenced by internal and external factors. Internal 
factors are the status of the motorcycle such as driver psychology, motorcycle position, steering angle, speed, etc. Meanwhile, external factors are environmental conditions such as the position of other vehicles, road borders, pedestrians, etc.

Modeling the behavior of the motorcycle is still being continuously developed, especially in developing countries which have the characteristics of mixed traffic. To get a better understanding of motorcycle behavior, one can look at maneuvers performed by drivers. Maneuver here can also be called as a movement performed by the motorcycle which is a part of motorcycle's movement to change a lane and also to avoid obstacles or slower vehicle in front of him.

Modeling the maneuver that can adequately simulate actual situations has many benefits, especially to solve problems in the field of transportation. Modeling of maneuvers can also describe how a driver interacts within a traffic system. In order to reach this goal, various methods have been developed to obtain a model that can adequately simulate actual situations. Based on the literature, several methods have been proposed for modeling vehicle movement, such as: Rule Based Model [6], Cellular Automata [7] [8] [9], and Social Force Model [10] [11] [12]. Each method has its own deficiencies and advantages. Rule Based Model is quite good at describing vehicle maneuvers in traffic but it only works well at low traffic density. Problems will occur when Rule Based Model is implemented on high-density traffic systems, making the simulation behave in an unrealistic manner. Celullar Automata (CA) describes vehicle maneuvers better than the rule-based system but has the disadvantage that its position updating rules are deterministic. Lately, Social Force Model (SFM) has been applied to describe vehicle maneuvers and it performed better than both Rule Based Model and Celullar Automata. SFM can describe the movement of vehicles based on a vector-based approach. In SFM, which includes a movement model and the decision-making process of the driver, vehicle behavior is described by the sum of several vector forces (acceleration force, repulsive force and attractive force). Although SFM can describe vehicle movement better than Rule Based or CA, this method does not input some parameters that have a large effect on achieving a realistic model, such as driver psychology or a probabilistic model for driver characteristics.

Markov Chain was broadly use for modeling traffic for different purposes, such as to reduce vehicle emission [13], short-term traffic flow forecasting [14], modeling the multi-traffic signal-control synchronization [15], traffic state prediction [16], travel time estimation [17], etc. However, no one has modeled the motorcycle maneuver using the Markov Chain model. In this research, we tried to build a model of motorcycle movement which only focused on maneuver action to avoid the obstacle along with the trajectories using a Markov Chain approach. In Markov Chain, the maneuver of motorcycle will described by state transition. The state transition model is depend on probability function which will use for determine what action will be executed next. The maneuver of motorcycle using Markov Chain model was validated by comparing the analytical result with the naturalistic data, with similarity is calculated using MSE. In order to know how good our proposed method can describe the maneuver of motorcycle, we try to compare the MSE of the trajectory based on Markov Chain model with those using polynomial approach that has been done in previous research [18].

This paper is organized as follows. In Section 2, modeling motorcycle maneuvers using a Markov chain process is presented. The simulation and analysis of applying this model are discussed in Section 3. Finally, in Section 4 this paper is concluded by stating that the Markov chain process can be implemented in modeling motorcycle movement trajectories for certain maneuvers.

\section{THE PROPOSED METHOD}

This research tries to build a model of maneuver behavior along with the trajectories using a Markov Chain approach. In a Markov Chain process, the driver will make decisions to determine what actions will be carried out in a certain environment. Vehicle $\alpha$ will be in a certain position, moving at a certain speed and steering angle at time $t$, denoted as motorcycle state $\alpha$ (internal information). Next, motorcycle $\alpha$ will receive external information from its environment, such as the positions and velocities of vehicles around it, for further processing to determine what action is to be taken by motorcycle $\alpha$. There are several steps must be executed to build a motorcycle maneuver model using Markov Chain: 1) Define set of states and actions, 2) Define the transition probability function, and 3) State mapping function.

\subsection{Set of States and Actions}

A state is the condition or status of motorcycle agent, which is a function of position $(x, y)$, speed $(v)$ and direction of steering maneuvers $(\theta)$. Meanwhile, action is described by a function of the maneuver $m \in$ 
$\{0,1,2\}$ with 0,1 , and 2 corresponds to no maneuver, right maneuver, and left maneuver respectively. Similarly, acceleration $a \in\{0,1,2\}$ with 0,1 , and 2 corresponds to fixed speed, acceleration, and deceleration.

In the Markov Chain process, the action of the motorcycle is determined by the probability of a transition states. There are ten states to describe the movement of motorcycle, along with nine actions. The set of actions $\left(A_{i}\right)$ and states $\left(S_{i}\right)$ that are used for the motorcycle maneuvers can be seen in Table 1 .

Table 1. Set of States and Actions

\begin{tabular}{l|l||ll}
\hline States & \multicolumn{1}{|c||}{ Definition } & Actions & \multicolumn{1}{c}{ Definition } \\
\hline$S_{0}$ & Vehicle with speed $0 \mathrm{~km} / \mathrm{h}$ & $A_{1}$ & Increases speed with no maneuver \\
\hline$S_{1}$ & Vehicle with speed $3 \mathrm{~km} / \mathrm{h}$ & $A_{2}$ & Increases speed with right maneuver \\
\hline$S_{2}$ & Vehicle with speed $6 \mathrm{~km} / \mathrm{h}$ & $A_{3}$ & Increases speed with left maneuver \\
\hline$S_{3}$ & Vehicle with speed $9 \mathrm{~km} / \mathrm{h}$ & $A_{4}$ & Decreases speed with no maneuver \\
\hline$S_{1 L}$ & Vehicle at a speed of $3 \mathrm{~km} / \mathrm{h}$ with left steering angle & $A_{5}$ & Decreases speed with right maneuver \\
\hline$S_{2 L}$ & Vehicle at a speed of $6 \mathrm{~km} / \mathrm{h}$ with left steering angle & $A_{6}$ & Decreases speed with left maneuver \\
\hline$S_{3 L}$ & Vehicle at a speed of $9 \mathrm{~km} / \mathrm{h}$ with left steering angle & $A_{7}$ & Decreases speed with right maneuver \\
\hline$S_{1 R}$ & Vehicle at a speed of $3 \mathrm{~km} / \mathrm{h}$ with left steering angle & $A_{8}$ & Fixed velocity with right maneuver \\
\hline$S_{2 R}$ & Vehicle at a speed of $6 \mathrm{~km} / \mathrm{h}$ with left steering angle & $A_{9}$ & Fixed velocity with left maneuver \\
\hline$S_{3 R}$ & Vehicle at a speed of $9 \mathrm{~km} / \mathrm{h}$ with left steering angle & & \\
\hline
\end{tabular}

\subsection{Transition Probabilities}

A maneuver is the movement to change the direction of a vehicle to move from one lane to another. Maneuvers in this study are influenced by several parameters, including the internal state of the vehicle as well as the external conditions, as shown in Figure 1.

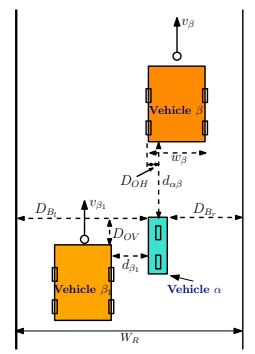

Figure 1. Illustration of Maneuver's Parameters

The parameter in Figure 1 are $d_{\alpha \beta}, v_{\beta}, w_{\beta}, d_{\beta_{1}}, d_{\beta_{2}}, \ldots, d_{\beta_{n}}, v_{\beta_{1}}, v_{\beta_{2}}, d_{\alpha B_{r}}, d_{\alpha B_{l}}, D_{O H}, D_{O V}$, and $W_{R}$. Where $d_{\alpha \beta}$ is a distance between motorcycle $\alpha$ and vehicle $\beta, v_{\beta}$ is speed of vehicle $\beta, w_{\beta}$ is the width of vehicle $\beta ;\left(d_{\beta_{1}}, d_{\beta_{2}}, \ldots, d_{\beta_{n}}\right)$, distance between motorcycle $\alpha$ and vehicles to the left and right, $v_{\beta_{1}}$ is a speed of vehicle to the left, $v_{\beta_{2}}$ is speed of vehicle to the right, $d_{\alpha B_{r}}$ is the distance between vehicle $\alpha$ and the right border, $d_{\alpha B_{l}}$, distance between vehicle $\alpha$ and the left border, $D_{O H}$ is a horizontal offset distance to objects that are in front of the vehicle, $D_{O V}$ is the vertical offset distance to vehicles to the left or the right of the vehicle, and $W_{R}$ is the width of road.

There are two steps performed to build the transition probability function. Firstly, to model the probability of maneuverability function of the rider. Secondly, to model the probability of maneuvering direction (left or right) to be taken by the motorcycle.

\subsubsection{Transition Probability of Vehicle Maneuvers}

The biggest factor that influenced the maneuver is if there is an obstacle in front of the motorcycle. As function to model the desire of maneuvering of the driver, negative exponential function can be used to obtain a great value for small distances and a small value for large distances. In addition, a critical distance factor $\left(D_{c}\right)$ should be added, which represents the critical distance where the motorcycle must do maneuver. If a maneuver 
cannot be carried out for example because there are vehicles to the right and left, then the action is decelerate the speed. The maneuver intention probability of motorcycle $\alpha$ stated as $\mathrm{P}_{\alpha}$ can be expressed as follows.

$$
\mathrm{P}_{\alpha}= \begin{cases}e^{-k_{1}\left(d_{\alpha \beta}-D_{c}\right)}, & \text { for } d_{\alpha \beta}>D_{c} \\ 1, & \text { for } d_{\alpha \beta} \leq D_{c}\end{cases}
$$

Where $d_{\alpha \beta}$ is the distance between motorcycle $\alpha$ and the vehicle in front of it (vehicle $\beta$ ), $D_{c}$ is represents the critical distance where the motorcycle must do maneuver or decelerate the speed, $k_{1}$ is a constant that shows an increased slope rate of a negative exponential curve as a function of the distance between the two vehicles. Physically, a small value of $k_{1}$ indicates that the driver wants to maneuver despite the distance to the vehicle in front still being quite large (alert driver), while a high value of $k_{1}$ indicates that the driver wants to maneuver when the distance to the vehicle in front is already very small (a driver who is reluctant to maneuver unless pressed).

\subsubsection{Probability Estimation of Maneuver Direction}

When the decision to maneuver based on $\mathrm{P}_{\alpha}$ exceeds a certain threshold value, the next step is modeled the optimum maneuver direction. The possible directions for the maneuver are determined by the condition of the road, the borders to the right and left, and other vehicles in the vicinity. In this subsection, we build maneuver direction probability model for six scenarios that commonly occur in traffic, as shown in Figure 2 . This scenario is still possible to be developed further in a follow-up study, which will be tailored to observations in the field and processing the data obtained. The derivation process of the probability model has been done in a previous research [18].

Table 2. Maneuver Direction Probabilities

\begin{tabular}{|c|c|}
\hline Scenario & Probability of Maneuver Direction \\
\hline There are no obstructions on the left nor right & $\begin{array}{l}\mathrm{P}_{\theta_{L}}=\mu \cdot e^{k_{3} \cdot \frac{\left(d_{\alpha B_{l}}-d_{C_{l}}\right)}{W_{R}}-k_{2} \cdot \gamma_{l}} \\
\mathrm{P}_{\theta_{R}}=\mu \cdot e^{k_{3} \cdot \frac{\left(d_{\alpha B_{r}}-d_{C_{r}}\right)}{W_{R}}-k_{2} \cdot \gamma_{r}}\end{array}$ \\
\hline There is an obstruction to the right of the motorcyle & $\begin{array}{l}\mathrm{P}_{\theta_{L}}=\mu \cdot e^{k_{3} \cdot \frac{\left(d_{\alpha B_{l}}-d_{C_{l}}\right)}{W_{R}}-k_{2} \cdot \gamma_{l}} \\
\mathrm{P}_{\theta_{R}}= \begin{cases}1-e^{-k_{4}\left(d_{\beta_{1}}-d_{\beta_{1 C}}\right)} & , \text { for } d_{\beta_{1}}>d_{\beta_{1 C}} \\
0 & , \text { for } d_{\beta_{1}} \leq d_{\beta_{1 C}}\end{cases} \end{array}$ \\
\hline There is an obstruction to the left of the motorcycle & $\begin{array}{l}\mathrm{P}_{\theta_{L}}= \begin{cases}1-e^{-k_{5}\left(d_{\beta_{1}}-d_{\beta_{1 C}}\right)} & , \text { for } d_{\beta_{1}}>d_{\beta_{1 C}} \\
0 & , \text { for } d_{\beta_{1}} \leq d_{\beta_{1 C}}\end{cases} \\
\mathrm{P}_{\theta_{R}}=\mu \cdot e^{k_{3} \cdot \frac{\left(d_{\left.\alpha B_{r}-d_{C_{r}}\right)} W_{R}\right.}{W_{R} \cdot \gamma_{r}}}\end{array}$ \\
\hline There are obstruction to the right and to the left of the motorcycle & 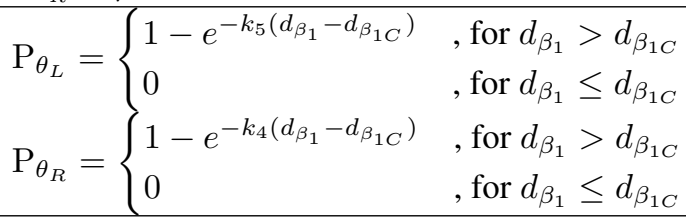 \\
\hline There is a border on the left or on the right & $\begin{array}{l}\mathrm{P}_{\theta_{L}}=0 \\
\mathrm{P}_{\theta_{R}}=1\end{array}$ \\
\hline There are boarders and obstructions & $\begin{array}{l}\mathrm{P}_{\theta_{L}}=\mu \cdot e^{k_{3} \cdot \frac{\left(d_{\alpha B_{l}}-d_{C_{l}}\right)}{W_{R}}-k_{2} \cdot \gamma_{l}} \\
\mathrm{P}_{\theta_{R}}= \begin{cases}1-e^{-k_{4}\left(d_{\beta_{1}}-d_{\beta_{1 C}}\right)} & , \text { for } d_{\beta_{1}}>d_{\beta_{1 C}} \\
0 & , \text { for } d_{\beta_{1}} \leq d_{\beta_{1 C}}\end{cases} \end{array}$ \\
\hline
\end{tabular}

Table 2 shows the maneuver direction probability function. $\mathrm{P}_{\theta_{L}}$ denotes the probability of a left direction maneuver and $\mathrm{P}_{\theta_{R}}$ denotes the probability of a right direction maneuver, with $k_{2}, k_{3}, k_{4}$ and $k_{5}$ as constants. Parameter $\gamma$ denotes as a function of horizontal offset distances to objects that are in front of motorcycle $\alpha\left(D_{O H_{l}}, D_{O H_{r}}\right)$, where $w_{\beta}=d_{O H_{l}}+d_{O H_{r}}, \gamma_{l}=\frac{D_{O H_{l}}}{D_{O H_{l}}+d_{O H_{r}}}$ and $\gamma_{r}=\frac{D_{O H_{r}}}{D_{O H_{l}}+D_{O H_{r}}}$. $d_{C_{r}}$ and $d_{C_{l}}$ denotes critical distance with motorcycle on the left and right. The $\mu$ value can be set to the value that describes the habits of maneuvering direction of Indonesian driver. 


\subsection{State-Mapping Function}

After the transition probability function were determined, then we need to build a mapping function which describes the state-action mapping of decision making behavior of driver. Mathematically, this is expressed by

$$
\mathrm{P}\left(S_{t+1} \mid S_{t}, S_{\beta_{1}}, S_{\beta}, S_{\beta_{3}}, \cdots, m, a\right)=\mathrm{P}\left(S_{i+1} \mid S_{t}, m, a\right)
$$

with $\mathrm{P}\left(S_{i+1} \mid S_{i}, m_{i}, a_{i}\right)$ denotes probability function of maneuver and acceleration at time $t$.
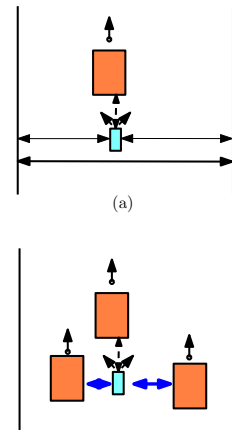

(d)

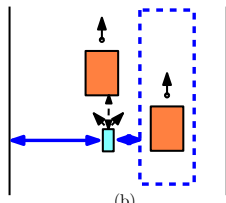

(b)

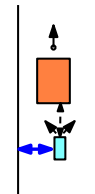

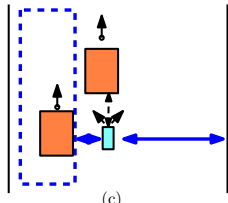

(c)

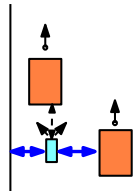

Figure 2. Traffic Scenario

\section{RESEARCH METHOD}

The research method consists of three main steps: 1) Data preprocessing, 2) Simulation, and 3) Comparing the simulation results with actual data. Data preprocessing was begin with doing the naturalistic observation using video streaming which ensures that the normal behavior can be observed and the data collected are not affected by the presence of researchers. Next, in order to obtained pixel coordinate which represents motorcycle's trajectory, coordinates transformation to convert the pixel coordinate to real-world coordinate as we seen on Figure 3. The preprocessing of data in this research is similar to those in our previous research [18].

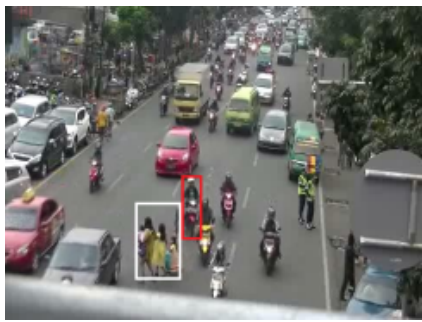

(a)

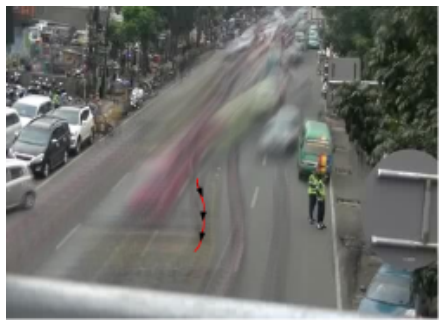

(b)

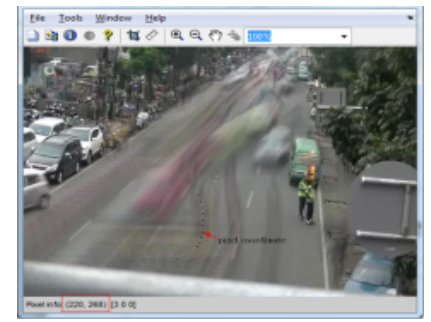

(c)

Figure 3. The flow of data preprocessing, (a) Targeted motorcycle; (b) Maneuvers trajectory; (c) Photo coordinate in a pixel

\section{SIMULATION RESULTS AND DISCUSSION}

The simulation results show that motorcycle movement modeling based on a Markov Chain process was successfully implemented. The Markov Chain model was evaluated by looking at a plotting diagram. There are three diagrams in Figure 4 which describes that Markov Chain was successfully implemented to modeled the manuever of motorcycle (a maneuver diagram, a state transition diagram, and an acceleration diagram). In Figure 4 (a), the changes of each state and speed for every vehicle during the simulation period can be seen clearly. The blue motorcycle change his state in order to get a savely driving. Besides, Figure 4 (c), we can see the behavior of the blue motorcycle making three times maneuver during simulation time, at the $5^{t h}$, the $19^{t h}$, and the $40^{t h}$ time step of the duration. In that time, the blue motorcycle sees a vehicle in front of 
him and try to avoid the vehicle by making a maneuver, while considering the border beside him to calculate the maneuver probability.

The performance of Markov Chain model is verified by comparing actual maneuver trajectory with the trajectory from Markov Chain model. Figure 5 (a) shows the actual trajectory data of ten motorcycle's that has taken independently at different time but plotted in one figure. It is interesting to note that there are different types of maneuver style in that trajectory, for example motorcycle 1 makes a complex maneuver pattern as to avoid the obstacle. In the other hand, motorcycle 3 and motorcycle 6 take a simple maneuver with considerable distance to complete this maneuver. Figure 5 (b) shows the track of Markov Chain model using

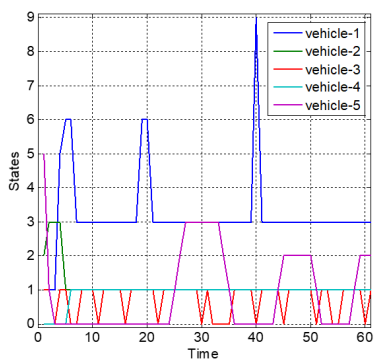

(a)

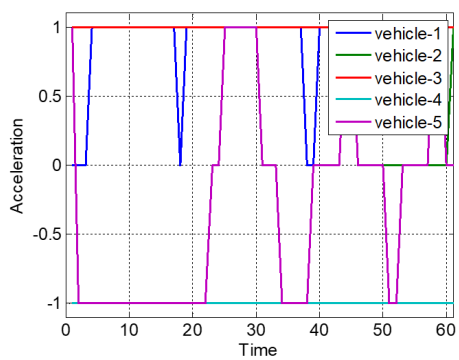

(b)

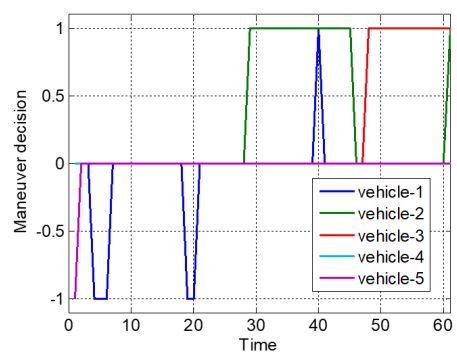

(c)

Figure 4. Markov Chain simulation, (a) State-transition diagram; (b) Acceleration diagram; (c) Maneuver decision diagram

that only accommodate one maneuvering angle which is $45^{\circ}$. We observed in this figure that Markov Chain model successfully follow the trend of actual maneuver but cannot produce a smooth pattern. To improve the situation, in Figure 5 (c), we add additional states of maneuver steering angle which are $22.5^{\circ}, 45^{\circ}, 62.5^{\circ}$ to accommodate a smoother trajectory pattern which is very close to the actual one. As a comparison we plot the curve fitting of the track using static polynomial fitting which are $2^{\text {nd }}$ order polynomial, $3^{\text {rd }}$ order polynomial, and $4^{\text {th }}$ order polynomial as shown in Figure 5 (d) (e) (f). In those figures, we observed that polynomial can fit the actual trajectory as far as the trajectory is short and simple curve such as the maneuver of motorcycle 2,4 , and 9. However, polynomial fitting has difficulty to track a complex maneuver such as the maneuver of motorcycle 1 . This polynomial fitting also cannot track the slight maneuver such as maneuver of motorcycle 7 and 10 . 


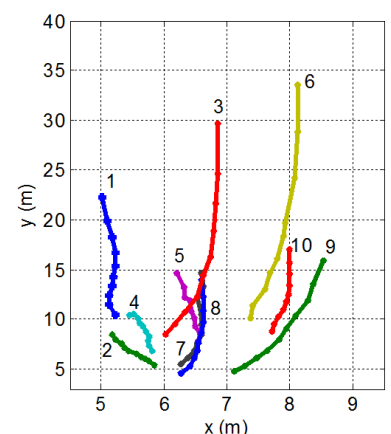

(a)

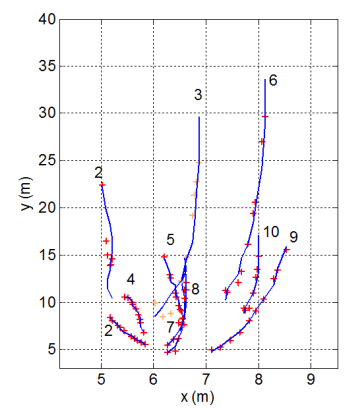

(d)

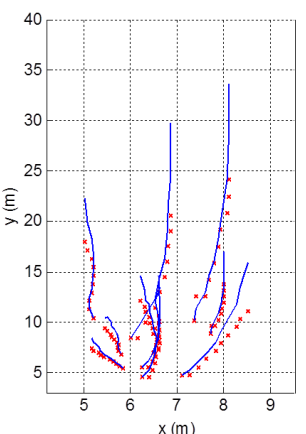

(b)

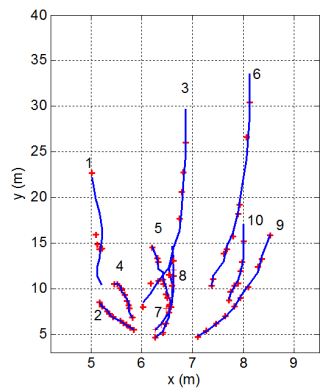

(e)

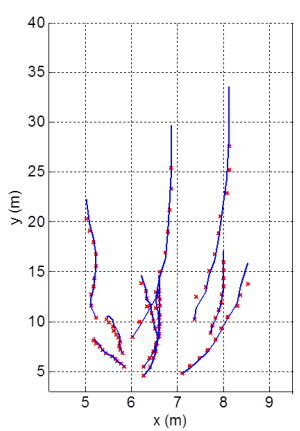

(c)

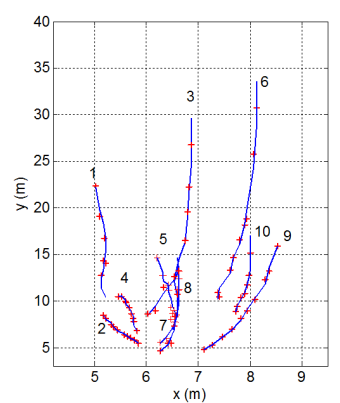

(f)

Figure 5. Manually track of ten different motorcycle's maneuver, (a) actual motorcycle's maneuver track (b) approximation of maneuver's track using Markov Chain with $\theta=45^{\circ}$ (c) approximation of maneuver's track using Markov Chain with $\theta=22.5^{\circ}, 45^{\circ}, 62.5^{\circ}(\mathrm{d})$ approximation of maneuver's track using $2^{\text {nd }}$ order polynomial (e) approximation of maneuver's track using $3^{\text {rd }}$ order polynomial (f) approximation of maneuver's track using $4^{\text {th }}$ order polynomial

Performance of each fitting method is shown in Table 3. In Table 3, we observed that Markov Chain model give the smallest MSE which 0.7666 about 0.24 better than $4^{\text {th }}$ order polynomial. Even though in most cases the Markov Chain model using 22.5, 45, 62.5 has better MSE but in particular case, for example in motorcycle 6 , the performance of this model is worse than polynomial fitting, since the maneuver is very complex.

Table 3. Mean Square Error of Motorcycle Maneuver Trajectory Tracking

\begin{tabular}{|c|c|c|c|c|c|}
\hline Vehicle & $2^{\text {nd }}$ order & $3^{\text {rd }}$ order & $4^{\text {th }}$ order & $\theta=\mathbf{4 5}^{\circ}$ & $\theta=\mathbf{2 2 . 5}^{\circ}, \mathbf{4 5}^{\circ} \mathbf{6 2 . 5}^{\circ}$ \\
\hline 1 & 2.7076 & 2.6887 & 2.1865 & 1.8073 & 0.6907 \\
\hline 2 & 0.0849 & 0.0640 & 0.0626 & 0.4998 & 0.1472 \\
\hline 3 & 1.0267 & 0.8822 & 0.8655 & 1.6302 & 0.4086 \\
\hline 4 & 0.2525 & 0.2521 & 0.2509 & 0.9108 & 0.4336 \\
\hline 5 & 0.8472 & 0.8092 & 0.6743 & 1.1847 & 0.2783 \\
\hline 6 & 1.7213 & 1.3810 & 1.2796 & 3.8066 & 2.3199 \\
\hline 7 & 1.9405 & 1.9405 & 1.9344 & 2.0455 & 0.6516 \\
\hline 8 & 1.4145 & 1.4056 & 1.3684 & 2.0102 & 0.5179 \\
\hline 9 & 0.2622 & 0.2022 & 0.1966 & 2.0889 & 0.7340 \\
\hline 10 & 2.1705 & 1.6028 & 1.2978 & 3.8687 & 1.4841 \\
\hline Average & $\mathbf{1 . 2 4 2 8}$ & $\mathbf{1 . 1 2 2 8}$ & $\mathbf{1 . 0 1 1 7}$ & $\mathbf{1 . 9 8 5 3}$ & $\mathbf{0 . 7 6 6 6}$ \\
\hline
\end{tabular}

\section{CONCLUSION}

In this research, a simple model based on a Markov Chain process was implemented to describe motorcycle maneuver using ten states and nine actions. Besides, the probability function of maneuver intention 
was introduced in six scenarios which integrated to the state-mapping function of Markov Chain Model. The maneuver of motorcycle using Markov Chain model was verified by comparing the analytical result with the naturalistic data which give small MSE. This methods also compare with static polynomial fitting to know how good is Markov Chain can describe a motorcycle maneuver movement. As a result, we found that MSE of Markov Chain model is smaller than static polynomial fitting approach.

\section{ACKNOWLEDGMENT}

The authors would like to thank the Indonesia Endowment Fund for Education (LPDP Indonesia) and the Indonesian Ministry of Religious Affairs for their financial support of this study.

\section{REFERENCES}

[1] N. Bellomo and C. Dogbe, "On the modeling of traffic and crowds: A survey of models, speculations, and perspectives," SIAM Review, vol. 53, no. 3, pp. 409-463, 2011. [Online]. Available: https://doi.org/10.1137/090746677

[2] R. Mardiati, N. Ismail, and A. Faroqi, "Review of microscopic model for traffic flow," ARPN Journal of Engineering and Applied Sciences, vol. 9, no. 10, pp. 1794-1800, October 2014.

[3] K. Gopalakrishna and S. Hariprasad, "Real-time fatigue analysis of driver through iris recognition," International Journal of Electrical and Computer Engineering (IJECE), vol. 7, no. 6, pp. 3306-3312, 2017.

[4] S. K. Venkata, "An intelligent online vehicle tyre pressure monitoring system," International Journal of Electrical and Computer Engineering, vol. 2, no. 3, p. 301, 2012.

[5] T. S. Gunawan, A. Mutholib, and M. Kartiwi, "Performance evaluation of automatic number plate recognition on android smartphone platform," International Journal of Electrical and Computer Engineering, vol. 7, no. 4, p. 1973, 2017.

[6] L. Chong, M. M. Abbas, A. M. Flintsch, and B. Higgs, "A rule-based neural network approach to model driver naturalistic behavior in traffic," Transportation Research Part C: Emerging Technologies, vol. 32, pp. 207 - 223, 2013. [Online]. Available: http://www.sciencedirect.com/science/article/pii/S0968090X12001210

[7] Y. Zhang and H. Duan, "Modeling mixed traffic flow at crosswalks in microsimulations using cellular automata," Tsinghua Science and Technology, vol. 12, no. 2, pp. 214-222, April 2007.

[8] J. X. Ding, H. J. Huang, and Q. Tian, "A mixed traffic flow model based on a modified cellular automaton in two-lane system," in 2009 International Joint Conference on Computational Sciences and Optimization, vol. 2, April 2009, pp. 124-126.

[9] Z. dong Zhang, Y. fang yang, W. Qi, A. Chariete, and X. xiang Lin, "A cellular automata traffic flow model considering bus lane changing behavior with scheduling parameters," Discrete Dynamics in Nature and Society, vol. 2015, p. 7 pages, januari 2015.

[10] T. Ming and J. Hongfei, "An approach for calibration and validation of the social force pedestrian model," 2011, kalibrasi dan Validasi Parameter pada SFM.

[11] D. N. HUYNH, M. BOLTZE, and A. T. VU, "Modelling mixed traffic flow at signalized intersectionusing social force model," Journal of the Eastern Asia Society for Transportation Studies, vol. 10, pp. 17341749, 2013.

[12] W. Zeng, H. Nakamura, and P. Chen, "A modified social force model for pedestrian behavior simulation at signalized crosswalks," Procedia - Social and Behavioral Sciences, vol. 138, pp. 521 - 530, 2014. [Online]. Available: http://www.sciencedirect.com/science/article/pii/S1877042814041536

[13] J. Sun, C. Li, J. Ding, J. Yang, and Z. Liu, "A markov chain based traffic flow control model for reducing vehicles' co 2 emissions," in Vehicular Electronics and Safety (ICVES), 2015 IEEE International Conference on. IEEE, 2015, pp. 250-255.

[14] S. Sun, G. Yu, and C. Zhang, "Short-term traffic flow forecasting using sampling markov chain method with incomplete data," in Intelligent Vehicles Symposium, 2004 IEEE. IEEE, 2004, pp. 437-441.

[15] J. B. Clempner and A. S. Poznyak, "Modeling the multi-traffic signal-control synchronization: A markov chains game theory approach," Engineering Applications of Artificial Intelligence, vol. 43, pp. 147-156, 2015.

[16] C. Antoniou, H. N. Koutsopoulos, and G. Yannis, "Traffic state prediction using markov chain models," in Control Conference (ECC), 2007 European. IEEE, 2007, pp. 2428-2435.

[17] J. Yeon, L. Elefteriadou, and S. Lawphongpanich, "Travel time estimation on a freeway using discrete 
time markov chains," Transportation Research Part B: Methodological, vol. 42, no. 4, pp. 325-338, 2008.

[18] R. Mardiati, B. R. Trilaksono, Y. S. Gondokaryono, and S. S. Sulaksono, "Motorcycles trajectory tracking model based on polynomial least-squares approximation," Advanced Science Letters, vol. 23, no. 5, pp. 4537-4541, 2017.

\section{BIOGRAPHY OF AUTHORS}

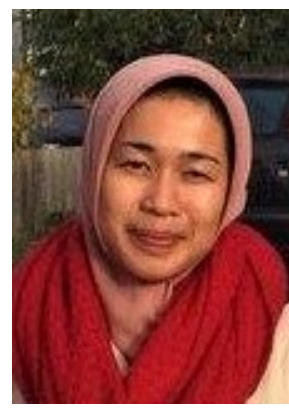

Rina Mardiati received her Bachelor degree in Mathematics Education from Universitas Pendidikan Indonesia (UPI) in 2006 and Master degree in Electrical Engineering from Institut Teknologi Bandung (ITB) in 2009. She is now a PhD student at the School of Electrical Engineering and Informatics, ITB. Her research is in the fields of mathematical modeling, simulation, traffic modeling and intelligent systems. She is affiliated with IEEE as a student member.

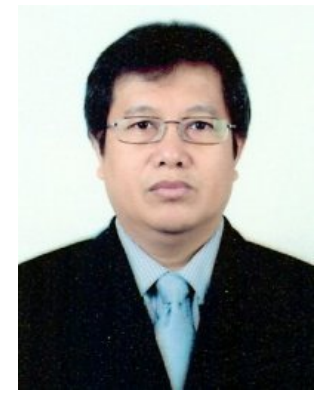

Bambang Riyanto Trilaksono received his Bachelor degree in Electrical Engineering from ITB, and Master and Doctorate degrees from Waseda University, Japan. He is now a professor at the School of Electrical Engineering and Informatics, ITB. His research interests include robust control, intelligent control \& intelligent systems, discrete event systems, control applications, telerobotics, embedded control systems, and robotics.

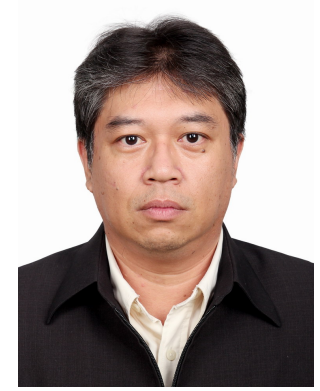

Yudi Satria Gondokaryono Gondokaryono received his Bachelor degree in Electrical Engineering from ITB, and Master and Doctorate degree from New Mexico State University. He is now an Assistant Professor in School of Electrical Engineering and Informatics at School of Electrical Engineering and Informatics, ITB. His research interests include human computer interaction, realtime, and embedded systems.

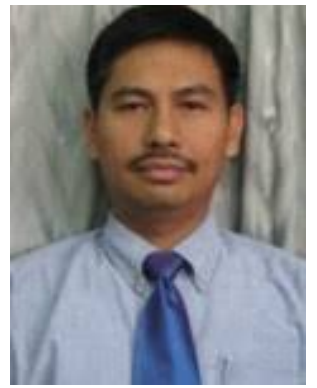

Sony Sulaksono Wibowo received his Bachelor and Master degrees in Civil Engineering from ITB, and Doctorate degree from Chualalangkorn University. He is now an assistant professor at the Department of Civil Engineering, ITB. His research interests include travel behavior, public transportation and transport planning, non-motorized transportation, transportation and the environment. 